\title{
Day 5:
}

\section{Preparing for the Future}




\title{
Exploring the Time Domain with Synoptic Sky Surveys
}

\author{
S. G. Djorgovski, A. A. Mahabal, A. J. Drake, M. J. Graham, \\ C. Donalek and R. Williams
}

California Institute of Technology, Pasadena, CA 91125, USA

email: george@astro.caltech.edu

Invited Talk

\begin{abstract}
Synoptic sky surveys are becoming the largest data generators in astronomy, and they are opening a new research frontier that touches practically every field of astronomy. Opening the time domain to a systematic exploration will strengthen our understanding of a number of interesting known phenomena, and may lead to the discoveries of as yet unknown ones. We describe some lessons learned over the past decade, and offer some ideas that may guide strategic considerations in the planning and execution of future synoptic sky surveys.
\end{abstract}

\section{Introduction: Exploring a New Domain}

In the 1990s astronomy transitioned from a data poverty to an immense, exponentiallygrowing data richness. The main agents of change were large digital sky surveys that produced data-sets measuring from a few to a few tens of Terabytes, and they, in turn, were enabled by burgeoning information technology. The challenge of the effective scientific use of such data-sets was met by the advent of the Virtual Observatory (VO) concept. The data volume continues doubling on a scale of $\sim 1-2$ years, reflecting Moore's law that describes the growth of the technology that produces the data. There is also an accompanying growth of data complexity and data quality. We are now transitioning into the Petascale regime, and the main agents of change are the synoptic sky surveys that cover large areas of the sky repeatedly. Some of the current surveys include CRTS, PTF and PanSTARS in the optical and various SKA prototypes in radio, leading to the next generation of facilities (such as LSST, SKA and many others described at this Symposium) that will effectively operate in a time-domain mode, producing tens of TB daily.

Time-domain astronomy (TDA) opens a new discovery space, not just by the sheer growth of data rates and data volumes but also by opening the "time axis" (actually, many axes) of the observable parameter space (OPS). A distinction should be made between the OPS, which is limited by our technology and the physical limitations of measurements (things like the transparency of the Earth's atmosphere or the ISM, diffraction limit, quantum limits, etc.) and the physical parameter space, which is populated according to the laws of nature; the mapping of one onto the other is not trivial. This expresses the vision of a systematic exploration of the OPS first formulated by Zwicky (1957), who referred to it as the "morphological box". History has shown that every time technology enables us to open a new portion of the OPS we are likely to discover some new types of objects and phenomena (Harwit 1975). Specifically, exploration of the time domain ("monitoring sky for variability") was eloquently advocated by Paczynski (2000).

It is a rich territory to explore. Some phenomena, such as various cosmic explosions, accretion or relativistic behaviour, can be studied only in the time domain. As a whole, 
TDA touches practically every field of astronomy, from the Solar System to cosmology and from stellar structure and evolution to extreme relativistic phenomena. Nor is it confined to the electromagnetic signals, as the neutrino and cosmic ray astronomy mature and gravitational-wave astronomy is born. This very richness makes the TDA a too diffuse concept, just as it makes little sense to talk about "spectroscopic astronomy" or "imaging astronomy". Rather, we can more meaningfully focus on the subjects of synoptic sky surveys or transient event discovery and characterization.

Recent discoveries of previously predicted phenomena such as supernova breakout shocks or tidal disruption events illustrate the scientific potential of TDA. It is reasonable to expect that a systematic exploration of the previously poorly covered parts of the OPS, in terms of the sensitivity, time cadences, area coverage, etc., may lead to a discovery of previously unknown phenomena.

TDA was also recognized as one of the most promising areas of the new, data-rich astronomy at the very onset of the VO concept (Djorgovski et al. 2001ab), and indeed it exercises every visualised VO functionality, and then some. As we argue below, a strong computational infrastructure is an essential enabling factor for the TDA.

\section{Some Lessons Learned}

The field is far too big to review adequately here. Our own experiences may be illustrative of the challenges involved, at least in the visible wavelength regime.

A search for highly variable and transient sources in the DPOSS plate overlaps (Mahabal et al. 2001; Granett et al. 2003) covered $\sim 8,000 \mathrm{deg}^{2}$ with at least 6 exposures ( 2 in each of 3 filters) and time base-lines ranging from a few months to $\sim 8$ years. We found that, at those time base-lines, roughly half of the high-amplitude variable objects are Galactic stars (mainly CVs and flaring dM), and half are AGNs (mostly blazars). We also found that in a single snapshot there will be $\sim 10^{3}$ optical transients per sky down to $\sim 20 \mathrm{mag}$, an estimate that has held well since then. It was clear that a variety of phenomena contribute to the population of optical transients (OTs), but that (near) real-time follow-up observations would be necessary to establish their nature.

The Palomar-Quest (PQ) survey (Djorgovski et al. 2008) and the concurrent NEAT project lasted $\sim 5$ years (ending in September 2008), with exploration of the time domain as the main science drivers. They resulted in discoveries of several hundred supernovæ $(\mathrm{SNe})$, mostly in collaboration with the LBNL Nearby Supernova Factory, studies of AGN variability, and studies of the most variable sources in the sky (aside from SNe) which again turned out to be mainly CVs and beamed AGN (Bauer et al. 2009). For the last 2 years of the survey we processed the drift scan observations in real time, the PQ Event Factory, which in some cases led to follow-up spectroscopy within an hour of the initial OT detection. The scientific returns were limited mainly by the poor quality of the data, and by the availability of follow-up. PQ was succeeded at the same telescope by the PTF (Rau et al. 2009), which operates with a very similar model but with a much better camera and much more abundant follow-up resources.

Aside from the confirmation that an OT event stream will contain a broad variety of astrophysical phenomena, several key lessons emerged. First, that asteroids are the main contaminant, with $\sim 10^{2}-10^{3}$ asteroids for each astrophysical transient, so a joint data processing and analysis is necessary. Secondly, that adequate follow-up - and in spectroscopy in particular - is essential for scientific returns; this is still a critical issue, and it is getting worse. Thirdly, that rapid classification of transients is essential in order to distill the incoming event stream down to a manageable number of interesting events worthy of the expenditure of the limited follow-up resources. A part of this requirement is a reliable and robust elimination of various data artifacts: in a massive data stream, 
inevitably there will be many glitches, and even the most unlikely things will happen, and most of them can look like transient events to a data pipeline. And finally, that the cost of software development will dominate any current or future synoptic sky survey, accounting perhaps for $\sim 80 \%$ of the total cost. One practical lesson was that the realtime processing demands must be accommodated in the overall system architecture, in addition to all that has been learned in the processing of single-epoch surveys.

We are currently conducting the Catalina Real-Time Transient Survey (CRTS; Drake et al. 2008, Djorgovski et al. 2011a, Mahabal et al. 2011). CRTS taps into a data stream used to search for NEO asteroids, thus both satisfying the need to separate asteroids from astrophysical OTs and illustrating yet again that the same data stream can feed many different scientific projects. CRTS has so far discovered $\sim 1,000$ SNe, including some novel or unusual types, a comparable number of CVs and dwarf novæ, variabilitybased IDs of previously unidentified Fermi gamma-ray sources, planets or other lowmass companions around white dwarfs, young stellar objects, and a plethora of variable stars and AGN (for example, Drake et al. 2010, 2011, 2012). CRTS imposes a very high detection threshold for OTs, and even this subset of the highest-amplitude events strains our follow-up capabilities. If we modify the pipeline to pick all statistically significant variables, the number of OTs would grow by at least an order of magnitude.

We are accumulating an unprecedented data-set of images and source catalogues (light curves) for $>5 \times 10^{8}$ sources covering $\sim 33,000 \mathrm{deg}^{2}$, spanning the time base-lines from $10 \mathrm{~min}$ to $\sim 7$ years and growing. This archival information is extremely useful for the interpretation of OTs, and it can enable a variety of archival TDA studies.

One lesson of CRTS is that a synoptic sky survey need not be photometric: its job is to discover transients, which can be done very efficiently in a single bandpass (or just an unfiltered CCD); the photometry is best done as a part of the follow-up. This relaxes many calibration and data quality demands faced by surveys that aim to be photometric. One should separate discovery of OTs from their characterization.

Another, iterated, lesson is that spectroscopic follow-up is already a key bottleneck, with only maybe $\sim 10 \%$ of CRTS transients followed. The problem will get worse by orders of magnitude with the next generation of synoptic sky surveys. Thus, the need for effective automated classification of transient events is critical.

\section{Cyber-Infrastructure for Time-Domain Astronomy}

TDA is by its nature very data-intensive, requiring a strong cyber-infrastructure that includes data processing pipelines, archiving, automated event classification and distribution, assembly of relevant information from the new data and the archives, etc.

The ephemeral nature of transient events requires that they are electronically distributed (published) in real time, in order to maximize the chances of a necessary followup. To this effect, we developed VOEvent, a VO-compliant standard for the event information exchange. Our vision was to lay the foundations for the robotic telescope networks with feedback, that would discover and follow-up transients, involving a variety of computational and archival data resources, and to facilitate event publishing, brokering, and interpretation. The next step was to develop a concept of event portfolios, that would automatically accumulate the relevant information and make it both machine- and human-accessible, via the web services and various electronic subscription mechanisms. The current implementation is SkyAlert (Williams et al. 2009).

The challenge of an automated event classification and follow-up prioritization is still outstanding. All OTs look the same when discovered - a star-like object that has changed its brightness significantly relative to the comparison base-line - and yet, they represent a vast range of different physical phenomena, some of which are more interesting than 
the others. Nowadays, surveys generate tens to hundreds of OTs per night; LSST may find $\sim 10^{5}$ to $10^{7}$ per night. Which ones are worthy of the expenditure of valuable and limited follow-up resources?

This problem entails some special challenges beyond traditional automated classification methods, which are usually done in some feature vector space with an abundance of homogeneous data. Here, the input information is generally sparse and heterogeneous, and often with a poor $S / N$; there are only a few initial measurements, and they differ from case to case with differing measurement errors; the contextual information is often essential and yet difficult to capture and incorporate in the classification process; many sources of noise, instrumental glitches, etc., can masquerade as transient events in the data stream; new, heterogeneous data arrive, and the classification must be iterated dynamically. The process must be automated, robust and reliable, with at most a minimal human intervention. Requiring high completeness (not missing any interesting events) and low contamination (a few false alarms), and the need to complete the classification process and make an optimal decision about expending valuable follow-up resources in (near) real time are substantial challenges that require some novel approaches (Donalek et al. 2008, Mahabal et al. 2008, Djorgovski et al. 2011b).

Most of the information about any given event initially, and often permanently, would be archival and/or contextual: spatial (what is around the event), temporal (what is its past light curve), and panchromatic (has it been detected at other wavelengths). Applying it may require a human (expert) judgment, and yet human involvement does not scale to the forthcoming event data streams. We are working on methods to harvest human pattern recognition skills and turn them into computable algorithms.

\section{Concluding Comments}

TDA - or simply astronomy with synoptic sky surveys - is intrinsically astronomy of telescope-computational systems. An optimal strategy may be to have dedicated survey telescopes, and surveys that are not overburdened by other requirements (e.g., multicolor photometry), and a hierarchy of follow-up facilities. For example, there may be a set of smaller, robotic telescopes providing multicolour photometry and helping to select the most promising events for spectroscopy. It would also make sense to coordinate surveys at different wavelengths to serve as a first-order mutual multi-wavelength follow-up.

There is an understandable trend to optimize a given survey's parameters (cadence, depth, etc.) for a given scientific goal such as SNe or NEO asteroids. That inevitably introduces selection biases against objects whose variability may not be captured well with that particular window function, thus diminishing the likelihood of truly novel discoveries. It would be good to have a broad spectrum of time base-lines that can capture a variety of phenomena, both known and as yet unknown. It would make sense if the competing surveys coordinate their sky coverage and cadences, and share the data.

Adequate and effective follow-up, especially optical spectroscopy, remains a key limiting factor. In a situation where there is a steady and abundant stream of events, the highly disruptive Target-of-Opportunity approach is not optimal; dedicated follow-up facilities are needed. The current-generation spectrographs on large telescopes tend to be optimized for highly multiplexed spectroscopy of faint objects, for example for studies of galaxy evolution. In contrast, the follow-up of transient events requires efficient single-object spectroscopy with relatively short exposures. Trying to repurpose existing equipment for a highly inefficient use makes little sense: telescopes and instruments dedicated for spectroscopic follow-ups of transient events should be designed accordingly.

All this has to be built on a strong cyber-infrastructure for data processing and archiving, event discovery, classification and publishing, etc. Automated, robust and reliable 
event classification is a key need for effective scientific returns and for optimal use of expensive facilities and resources. Given the importance of archival data for the early classification and interpretation of events, efficient, VO-type data services will be increasingly more important. Overall, a strong investment in astroinformatics, including facilities, software, and scientist training, is a major strategic need.

A transition from a data poverty regime to data overabundance will also change the astronomical sociology and operational modes: we are already in a situation where the producers of these massive data streams cannot fully exploit them in a timely manner. Thus, the focus of value shifts from the ownership of data to the ownership of expertise needed to make the discoveries. A key concept, promoted by the CRTS, is the completely open data philosophy: making the synoptic sky survey and event data streams available immediately to the world. While that trend was already apparent with the single-epoch surveys, it becomes critical with the synoptic sky surveys and the highly perishable transient events that they discover. As the data rates exceed the capabilities of any individual group to follow up effectively, it only makes sense to open them up, and thus engage a much broader segment of the astronomical community; in fact, it would be irresponsible to do otherwise. While the concept of a proprietary data period may still make sense for some types of targeted observations, it does not do so for the exponentially growing data streams of today or the future.

Finally, perhaps real-time astronomy with OTs is being overemphasized. There is a lot of excellent, non-time-critical science that can be done with the growing archives from synoptic sky surveys, such as a systematic search for variables of given types (like the RR Lyraes for mapping the Galactic structure), improved characterization of AGN variability as a constraint on theoretical models of accretion and beaming, etc.

Acknowledgments: This work was supported in part by the NASA grant 08-AISR080085, and the NSF grants AST-0407448, AST-0909182 and IIS-1118041. We thank numerous collaborators from the DPOSS, PQ, and CRTS surveys, and the VO and astroinformatics communities for the work and ideas that have shaped our own ideas and scientific results. We also thank the organizers for a most interesting Symposium.

\section{References}

Bauer, A., et al. 2009, ApJ, 705, 46

Djorgovski, S. G., et al. 2001a, ASPCS, 225, 52

Djorgovski, S. G., et al. 2001b, in Mining the Sky, eds. A. Banday et al., ESO Astroph. Symp., p. 305, Berlin: Springer-Verlag

Djorgovski, S. G., et al. 2008, AN, 329, 263

Djorgovski, S. G., et al. 2011a, in: eds. T. Mihara \& N. Kawai (eds.), The First Year of MAXI: Monitoring Variable X-ray Sources (Tokyo: JAXA Special Publ.), in press

Djorgovski, S. G., et al. 2011b, in: A. Srivasatava, N. V. Chawla and A. S. Perera (eds.), CIDU Conf. 2011, (NASA), p. 174.

Donalek, C., et al. 2008 , AIPC, 1082, 252

Drake, A. J., et al. 2009, ApJ, 696, 870

Drake, A. J., et al. 2010, ApJ, 718, L127

Drake, A. J., et al. 2011, ApJ, 738, 125

Drake, A. J., et al. 2012, ApJ, submitted (arXiv/1009.3048)

Granett, B., et al. 2003, BAAS, 35, 1300

Harwit, M. 1975, QJRAS, 16, 378

Mahabal, A. A., et al. 2001, BAAS, 33, 1461

Mahabal, A. A., et al. 2008, AN, 329, 288

Mahabal, A. A., et al. 2011, BASI, 39, 387 
Paczyński, B. 2000, PASP, 112, 1281

Rau, A. et al. 2009, PASP, 121, 1334

Tonry, J. 2011, PASP, 123, 58

Williams, R., et al. 2009, ASPCS, 411, 115

Zwicky, F. 1957, Morphological Astronomy, (Berlin: Springer-Verlag)

\section{Appendix: A Figure of Merit for Synoptic Sky Surveys}

It has become customary to compare surveys using the etendue, a product of the telescope collecting area $A$ and the instrument field of view $\Omega$ as a figure of merit (FoM). However, $A \Omega$ simply characterizes the telescope and partly the instrument, and says nothing about the survey, such as its depth, coverage rate, cadence, etc. A more meaningful FoM is needed; see Tonry (2011) for a relevant discussion.

We propose the following indicator of a survey's discovery potential, a product of its spatio-temporal coverage rate, $C$, and the estimate of the depth, $D$, that may be reasonably expressed as proportional to the $S / N$ ratio of the individual exposures. Thus:

$$
C=R \times N_{p} \times f_{\text {open }} \quad \text { and } \quad D=\left[A \times t_{\text {exp }} \times \epsilon\right]^{1 / 2} / F W H M \sim S / N
$$

where $R$ is the area coverage in $\mathrm{deg}^{2} /$ night (not counting repeated exposures), $N_{p}$ is the number of passes per field in a given night, $f_{\text {open }}$ is the fraction of open time averaged over the year, including weather losses, engineering time, deliberate closures, etc., $A$ is the effective collecting area in $m^{2}, t_{\text {exp }}$ is the average exposure time in sec, $\epsilon$ is the overall efficiency (throughput) of the instrument, and FWHM is the typical seeing FWHM in arcsec. Clearly, all these parameters should be taken as typical or averaged over a year. Note that $f_{\text {open }}$ and $F W H M$ characterize the site, $A$ and $\epsilon$, and partly $R$, characterize the telescope+instrument, and the remaining parameters reflect the chosen survey strategy.

$C D$ represents a FoM for a discovery rate of events, so the nett discovery potential of a given survey would be $C D$ multiplied by the number of years the survey operates. While this FoM accounts for most of the important survey parameters, it still does not capture factors such as the sky background and transparency, the total number of sources detected (which clearly depends strongly on the Galactic latitude), the cadence, bandpasses, angular resolution, etc.; nor does it account for operational parameters such as the data availability, the time delay between observations and the event publishing, etc. Nevertheless, we believe that $C D$ is a much more relevant FoM than the traditional (and often mis-used) $A \Omega$, as far as a characterization of surveys is concerned.

The following table shows the estimated values of the relevant parameters and $C D$ for the 3 components of the CRTS, and for several other current or future surveys. The assumed values of input parameters are based on our own experience or on published values, and may be consistently too optimistic. The values of $C D$ are no better than $\sim 20 \%$.

\begin{tabular}{|l|c|c|c|c|c|c|c|c|c|c|}
\hline Survey & $R$ & $N_{p}$ & $f_{\text {open }}$ & \multicolumn{1}{|c|}{$A$} & $t_{\text {exp }}$ & $\epsilon$ & $F W H M$ & $\mathbf{C}$ & $\mathbf{D}$ & CD \\
\hline CRTS:CSS & 1200 & 4 & 0.7 & 0.363 & 30 & 0.7 & 3 & 3360 & 0.92 & 3090 \\
CRTS:MLS & 200 & 4 & 0.7 & 1.767 & 30 & 0.7 & 3 & 560 & 2.03 & 1140 \\
CRTS:SSS & 800 & 4 & 0.7 & 0.196 & 20 & 0.7 & 3 & 2240 & 0.55 & 1240 \\
CRTS total & 2200 & 4 & 0.7 & $2.326)$ & & 0.7 & 3 & 6160 & & 5470 \\
PTF & 1000 & 2 & 0.7 & 1.131 & 60 & 0.7 & 2 & 1400 & 3.45 & 4820 \\
SkyMapper & 800 & 2 & 0.7 & 0.785 & 60 & 0.8 & 2 & 1120 & 3.07 & 3440 \\
PS1 & 1000 & 4 & 0.7 & 2.54 & 30 & 0.8 & 1 & 2800 & 7.81 & 21860 \\
LSST & 5000 & 2 & 0.75 & 34.9 & 15 & 0.8 & 0.8 & 7500 & 25.6 & 192000 \\
\hline
\end{tabular}

